\title{
Truncation in Millimeter and Submillimeter-Wave Optical Systems
}

\author{
J. Anthony Murphy, Member, IEEE, Aidan Egan, and Stafford Withington, Member, IEEE
}

\begin{abstract}
We present a technique for determining the power that is lost when the field in a beam waveguide is truncated by an axially-symmetric stop. The technique is based on the principles of multimode Gaussian optics. Although the underlying theory is applicable to any long-focal-length optical system, we concentrate on beam waveguides that are fed by diagonal horns, corrugated horns, smooth-walled conical horns, and uniformlyilluminated apertures. The results are summarized in a series of plots which show truncation loss as a function of normalised aperture size and phase slippage. These plots can be used together with single-mode design techniques to minimize the size of millimeter and submillimeter-wave optical systems.
\end{abstract}

\section{INTRODUCTION}

$\mathrm{W}$ HEN designing millimeter and submillimeter-wave optical systems, it is necessary to know at what radius the propagating beam can be truncated without incurring a significant amount of loss. If the beam is produced by a free-standing antenna, the loss is easy to calculate, because the far-field power pattern of the antenna can be simply integrated over the region defined by the stop. If however, the beam has been refocused a number of times, perhaps by a series of lenses and mirrors, the loss is more difficult to calculate, because it is necessary to know the form and size of the beam at the plane of interest, and these can only be determined directly if one knows the amplitude and the phase of the field at some other plane in the system. In this paper, we present a two-parameter technique for determining the power that is lost when the beam in a beam waveguide is truncated by an axially-symmetric stop; we assume that the amplitude and phase of the field across the aperture of the illuminating antenna are known.

To first order, a known field can be traced through a long-focal-length optical system by extracting the lowestorder Gaussian mode [1]. In the case of a corrugated horn, this approach is particularly attractive, since the field at the mouth of the horn is linearly polarized and can be described as a simple Gaussian to high accuracy [2]; truncation can then be avoided by ensuring that the diameters of the optical components and stops making up the system are greater than 6 times the local $1 / e$ Gaussian radius [3]. In the case of a diagonal or smooth-walled

Manuscript received August 5, 1992; revised May 17, 1993.

J. A. Murphy and A. Egan are with Department of Experimental Physics, Maynooth College, Co. Kildare, Ireland.

S. Withington is with Cavendish Laboratory, University of Cambridge, CB3 OHE, England. conical horn, or indeed any antenna that does not have a Gaussian aperture distribution, the situation is more complicated, because although the simple Gaussian gives an indication of how the scale size of the beam changes as it passes through an optical system, it gives no information about how the form of the beam changes [4], [5]. This limitation is particularly troublesome when the illuminating antenna has a highly-truncated aperture field, because then the beam changes from being spatially confined to being spatially diffuse as one moves in and out of foci. In the single-mode approximation, one is therefore left with the problem of surmising how the $1 / e$ Gaussian radius is related to the poorly-defined radial edge of the actual beam.

To solve this problem higher-order modes can be introduced into the theoretical description of the propagating field - the use of multimode Gaussian optics to analyse millimeter and submillimeter-wave optical systems was first suggested by Martin [6], and later extended by Wylde [2], Lamb [7], and Padman [3]. At first sight, this extension seems to complicate beyond usefulness the simple picture provided by the single-mode approximation. It should be appreciated, however, that in the multimode case, the scale size and form of the beam at a plane are completely characterized by the Gaussian radius and phase slippage, respectively. Thus, the field at a stop, and consequently the truncation loss, are completely characterized by two parameters, both of which are easily calculated using single-mode design techniques. The most involved part of an analysis lies in plotting contours of constant loss as a function normalized truncation and phase slippage for the particular type of horn being used.

In Section II, we summarize how antenna fields are expanded in terms of associated-Laguerre Gaussian (ALG) modes, and we show how these expansions can be used to plot contours of constant loss as a function normalized truncation radius and phase slippage. In Section III, we consider in detail the cases of diagonal, smooth-walled conical, corrugated, and uniformly-illuminated horns. Finally, in Section IV, we describe how these plots can be used together with single-mode design techniques to calculate the loss at any plane in a complicated quasioptical system: This section is self-contained and can be used without reference to other parts of the paper.

\section{Analysis of Truncation Using ALG Modes}

For a circular stop that is coaxial with the direction of propagation and perfectly absorbing outside of the trans- 
mitting region, the propagating beam is most conveniently described as a sum of associated Laguerre-Gaussian (ALG) modes [8]:

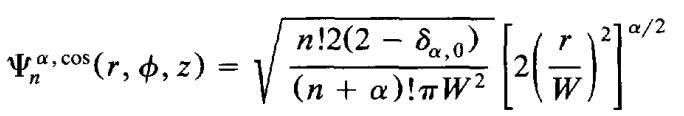

$$
\begin{aligned}
& \cdot L_{n}^{\alpha}\left(2\left(\frac{r}{W}\right)^{2}\right) \cos (\alpha \phi) \\
& \cdot \exp \left[-\left(\frac{r}{W}\right)^{2}-j k\left(z+\frac{r^{2}}{2 R}\right)+j \psi_{n \alpha}\right], \\
& \Psi_{n}^{\alpha, \sin }(r, \phi, z)=\sqrt{\frac{n ! 4\left(1-\delta_{\alpha, 0}\right)}{(n+\alpha) ! \pi W^{2}}}\left[2\left(\frac{r}{W}\right)^{2}\right]^{\alpha / 2} \\
& \cdot L_{n}^{\alpha}\left(2\left(\frac{r}{W}\right)^{2}\right) \sin (\alpha \phi) \\
& \cdot \exp \left[-\left(\frac{r}{W}\right)^{2}-j k\left(z+\frac{r^{2}}{2 R}\right)+j \psi_{n \alpha}\right]
\end{aligned}
$$

where $r$ is the radial distance from the beam axis (the $z$ axis), $\phi$ is the azimuthal angle, and $L_{n}^{\alpha}$ is an associated Laguerre polynomial of order $n$ and degree $\alpha$, where both $n$ and $\alpha$ are integers greater than or equal to zero. In these equations, $W=W(z)$ is the $1 / e$ Gaussian radius and $R=R(z)$ the phase-front radius of curvature; both of these are functions of the distance $z$ from the commonmode waist. $\psi_{n \alpha}=(2 n+\alpha+1) \psi_{0}(z)$ is the phase slippage between the waist and the plane of interest, and $\psi_{0}(z)=\tan ^{-1}\left(\pi W^{2} / \lambda R\right)$ is the phase slippage for the fundamental mode [8]. As stated in the Introduction, the scale size of the beam at a plane is characterized by the 1 / $e$ Gaussian radius $W(z)$, and the form of the beam is characterized by the phase slippage $\psi_{0}(z)$. Notice that the associated Laguerre polynomials, $L_{n}^{\alpha}$, are defined such that the generalized power in each mode $\iint\left|\Psi_{n}^{\alpha}(r, \phi)\right|^{2}$ $r d r d \phi$ is unity.

We can expand the co-polar and cross-polar components of a beam in the form

$$
\begin{aligned}
\Psi(r, \phi, z)=\sum_{n=0}^{\infty} \sum_{\alpha=0}^{\infty} A_{n \alpha} \Psi_{n}^{\alpha, \cos }(r, \phi, z) & \\
& \quad+\sum_{n=0}^{\infty} \sum_{\alpha=1}^{\infty} B_{n \alpha} \Psi_{n}^{\alpha, \sin }(r, \phi, z) .
\end{aligned}
$$

In reality, we truncate the series once the power in the sum is close to $100 \%$ of that in the actual beam. To determine the mode coefficients $A_{n \alpha}$ and $B_{n \alpha}$, we evaluate the overlap integrals at the aperture of the horn: This procedure is described in detail in [9].
If a beam is truncated at plane $z$ on the optical axis then the fraction of the total power transmitted is given by

$$
\begin{aligned}
P_{t r}= & \frac{\int_{r=0}^{r_{t}} \int_{\phi=0}^{2 \pi} E \cdot E^{*} r d r d \phi}{\int_{r=0}^{\infty} \int_{\phi=0}^{2 \pi} E \cdot E^{*} r d r d \phi} \\
= & \frac{\int_{r=0}^{r_{t}} \int_{\phi=0}^{2 \pi}\left|\Psi_{\mathrm{co}}(z)\right|^{2}+\left|\Psi_{\mathrm{cs}}(z)\right|^{2} r d r d \phi}{\int_{r=0}^{\infty} \int_{\phi=0}^{2 \pi}\left|\Psi_{\mathrm{co}}(z)\right|^{2}+\left|\Psi_{\mathrm{cs}}(z)\right|^{2} r d r d \phi},
\end{aligned}
$$

where co and cs denote the co-polar and cross-polar fields, respectively, and $r_{t}$ is the radius of the truncating stop. In the following discussion, the co-polar and crosspolar fields $\Psi_{\mathrm{co}}, \Psi_{\mathrm{cs}}$ have been normalized to make the total generalized power $\int_{r=0}^{\infty} \int_{\phi=0}^{2 \pi} \boldsymbol{E} \cdot \boldsymbol{E}^{*} r d r d \phi$ unity. The above equation can be expressed in terms of the phase slippage $\Delta \psi_{0}$ between the aperture of the horn (where the mode coefficients $A_{n \alpha}, B_{n \alpha}$ were evaluated) and the plane of interest:

$$
\begin{aligned}
\left(P_{\mathrm{tr}}\right)_{\mathrm{co}, \mathrm{cs}}= & \int_{0}^{x_{t}} d x \sum_{m, n, \alpha}^{\infty}\left(A_{m \alpha} A_{n \alpha}+B_{m \alpha} B_{n \alpha}\right)_{\mathrm{co}, \mathrm{cs}} \\
& \cdot \frac{x^{\alpha} L_{m}^{\alpha}(x) L_{n}^{\alpha}(x) \exp [-x]}{\sqrt{(m+\alpha) !(n+\alpha) ! / m ! n !}} \\
& \cdot \exp \left[2(n-m) j \Delta \psi_{0}\right] \\
= & \sum_{m, n, \alpha}^{\infty}\left(A_{m \alpha} A_{n \alpha}+B_{m \alpha} B_{n \alpha}\right)_{\mathrm{co}, \mathrm{cs}} I_{m, n}^{\alpha}\left(x_{t}\right) \\
& \cdot \exp \left[2(n-m) j \Delta \psi_{0}\right]
\end{aligned}
$$

where $x_{t}=2\left(r_{t} / W\right)^{2}$ and

$$
I_{m, n}^{\alpha}\left(x_{t}\right)=\int_{0}^{x_{t}} \frac{x^{\alpha} L_{m}^{\alpha}(x) L_{n}^{\alpha}(x) \exp [-x]}{\sqrt{(m+\alpha) !(n+\alpha) ! / n ! m !}} d x .
$$

To be consistent, we note that $B_{n, 0}$ are all zero. The subscripts co and cs denote the co-polar and cross-polar fields, respectively, and the total power transmitted $P_{\mathrm{tr}}$ is the sum of the two components.

A key feature of the proposed technique, is that it is possible to derive recursion relationships for the integrals $I_{m, n}^{\alpha}\left(x_{t}\right)$, which enable the truncation calculations to be evaluated easily. The relationships are

$$
\begin{gathered}
I_{m+1, n+1}^{0}=I_{m, n}^{0}+\left(L_{m}\left(x_{t}\right)-L_{m+1}\left(x_{t}\right)\right) \\
\cdot\left(L_{n+1}\left(x_{t}\right)-L_{n}\left(x_{t}\right)\right) e^{-x_{t}}, \\
\sqrt{\alpha+1} I_{m, 0}^{\alpha+1}=\sqrt{m+\alpha+1} I_{m, 0}^{\alpha}-\sqrt{m+1} I_{m+1,0}^{\alpha} \\
\text { for } m>0, \\
\sqrt{n+\alpha+1} I_{m, n}^{\alpha+1}=\sqrt{n} I_{m, n-1}^{\alpha+1}+\sqrt{m+\alpha+1} I_{m, n}^{\alpha} \\
-\sqrt{m+1} I_{m+1, n}^{\alpha} \text { for } m, n>0 .
\end{gathered}
$$


These relationships follow from combining two recursion relationships for associated Laguerre polynomials,

$$
\begin{gathered}
L_{n}^{\alpha}=L_{n}^{\alpha+1}-L_{n-1}^{\alpha+1}, \\
x L_{m}^{\alpha+1}=(m+\alpha+1) L_{m}^{\alpha}-(m+1) L_{m+1}^{\alpha},
\end{gathered}
$$

to yield the following recursion relationship for the product $L_{m}^{\alpha} L_{n}^{\alpha}$ :

$$
\begin{aligned}
x L_{m}^{\alpha+1} L_{n}^{\alpha+1}=x L_{m}^{\alpha+1} L_{n-1}^{\alpha+1}+(m & +\alpha+1) L_{m}^{\alpha} L_{n}^{\alpha} \\
& -(m+1) L_{m+1}^{\alpha} L_{n}^{\alpha} .
\end{aligned}
$$

To initiate the above series we need to compute $I_{n, 0}^{0}(=$ $\left.I_{0, n}^{0}\right)$, and these can be determined by using

$$
\begin{gathered}
I_{0,0}^{0}=1-\exp \left[-x_{t}\right], \\
I_{0, n}^{0}=\left(L_{n-1}\left(x_{t}\right)-L_{n}\left(x_{t}\right)\right) \exp \left[-x_{t}\right] .
\end{gathered}
$$

Note that, if we wish to truncate the series at $I_{m_{\max }}^{\alpha_{\max }, n_{\max }}$, then the last of the recursion relationships above requires determining $I_{m, n}^{0}$ up to $I_{m_{\max }+\alpha_{\max }, n}, I_{m, n}^{1}$ up to $I_{m_{\max }+\alpha_{\max }-1, n}$, and $I_{m, n}^{2}$ up to $I_{2 m_{\max }^{2}+\alpha_{\max }-2, n}$, etc.

It is clear that $P_{\mathrm{tr}}$ depends not only on the ratio of the truncation radius $r_{t}$ to the Gaussian beam width $W(z)$, but also on the phase slippage $\Delta \psi_{0}(z)$. As stated earlier, the Gaussian radius characterises the scale size of the beam at a plane and the phase slippage characterises the form of the beam; hence, one would expect the fraction of the total power transmitted to depend on these two quantities.

\section{RESULTS FOR DIFFERENT HORNS}

The determination of the Gaussian beam mode coefficients for the different horn types is covered in the literature: the corrugated horn in considered by Wylde [2], the conical horn by Murphy [4], the diagonal horn by Withington [5], and the uniformly illuminated aperture by Padman [3]. These calculations are considered in more detail in [9]. The most difficult field to propagate through an optical system is that produced by a uniformlyilluminated aperture. Although one cannot of course manufacture such a horn, it is precisely this field that would have to pass unimpeded through an optical system if one were trying to maximise the aperture efficiency of a large reflecting antenna; it is, therefore, interesting to consider this extreme example.

In order to parametrise our results for a given type of horn, we need to choose the Gaussian radius at the aperture $W_{h}$. There is a complete set of orthonormal modes associated with every value of $W_{h}$, and so we are free to choose $W_{h}$ in whatever way we wish. It has become common practice to use the value of $W_{h}$ that maximises the power in the lowest order mode; we will call this value $W_{h, \text { opt }}$. It can be shown, however, that in general $W_{h \text {, opt }}$ does not lead to a mode set that is particularly good at sampling the aperture field. In our loss calculations, we therefore used the value of $W_{h}$ that maximises the power in a finite number of modes [10]; once the contour plots were generated for this mode set, we then transformed
TABLE I

THE Size OF THE BEAM AT THE MOUTH OF THE HORN that Maximizes the Power In the Fundamental. Mode. For Feeds Having a CIRcular APERTURe, $a$ IS THE RADIUS OF THE APERTURE, WHEREAS For Feeds Having a SQUARE APERTURe, $a$ IS THE SiDELENGTH OF THE APERTURE.

\begin{tabular}{ll}
\hline Horn Type & $W_{h, \text { opt }}$ \\
\hline Top-hat & $0.892 a$ \\
Corrugated conical & $0.644 a$ \\
Smooth-wall conical & $0.770 a$ \\
Diagonal pyramidal & $0.430 a$ \\
\hline
\end{tabular}

back to the more commonly-used mode set based on $W_{h \text { opt }}$ for design purposes. The algebra associated with this transformation is given in [9]. The values of $W_{h \text {, opt }}$ used are listed in Table I. In the discussion that follows it is assumed that the beam radii $W$ are based on $W_{h \text {, opt }}$, and we drop the subscript opt for convenience.

In Figs. 1-4 we show, for the diagonal horn, smoothwalled conical horn, corrugated conical horn, and uniformly-illuminated aperture, contours of constant loss as a function of normalized truncation radius $r_{t} / W$ and differential phase slippage $\Delta \psi_{0}$.

It can be seen that for $\Delta \psi_{0}$ close to zero, the fields are spatially confined; whereas for $\Delta \psi_{0}$ close to $\pi / 2$, the fields are diffuse. For a diffraction-limited horn, these values correspond to positions in the optical system where there are images of the aperture and Fourier transforms of the aperture, respectively. It is important to realize that the central portion of this plot does not necessarily correspond to the beam waist. In fact, in the case of a physically realizable horn, the waist occurs at some negative phase slippage, and the phase slippage only becomes zero once the diverging beam has reached the aperture. As the beam propagates away from the aperture, sidelobe structure develops, and a spatial redistribution of power occurs as the image of the horn undergoes diffraction. This phenomenon shows up as $P_{\mathrm{tr}}$ developing off-axis wings with increasing values of $\Delta \psi_{0}$. The majority of the power still remains within $r=W$, emphasizing that the simple Gaussian gives a reasonable first-order description of the diffraction process.

If one is interested in the extent of the far-field beam, then the corresponding $\Delta \psi_{0}$ depends on the phase error $\left(s=a^{2} / 2 \lambda L\right)$ across the mouth of the horn. If the phase error is zero, then the waist occurs at the aperture, and the far-field beam pattern corresponds to $\Delta \psi_{0}=\pi / 2$. If there is a finite phase error, the waist is not at the aperture, and the far-field power pattern corresponds to a $\Delta \psi_{0}$ of less than $\pi / 2$ [11]. In general, if the beam propagates some distance through a waveguide before reaching the point at which it is truncated, the phase slippage corresponds to the total phase slippage accumulated since leaving the aperture. Notice that because $\Delta \psi_{0}$ is multiplied by an even number when calculating power loss, $P_{\text {tr }}$ will be the same for $\Delta \psi_{0}$ and $\Delta \psi_{0}+n \pi$, where $n$ is an integer. $P_{\mathrm{tr}}$ is therefore only plotted for values of $\Delta \psi_{0}$ lying between $-\pi / 2$ and $\pi / 2$. Strictly speaking only half 


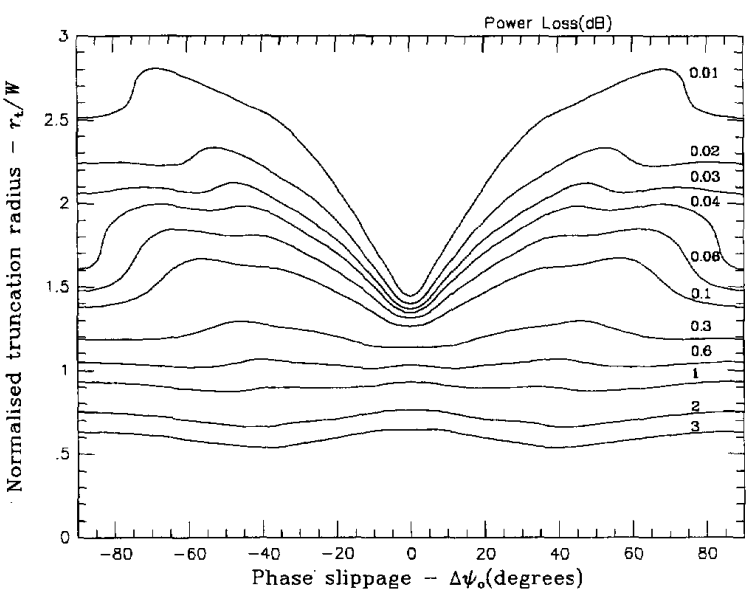

Fig. 1. Contours of constant power loss $(\mathrm{dB})$ as a function of phase slippage and normalized truncation for the beam of a corrugated horn.

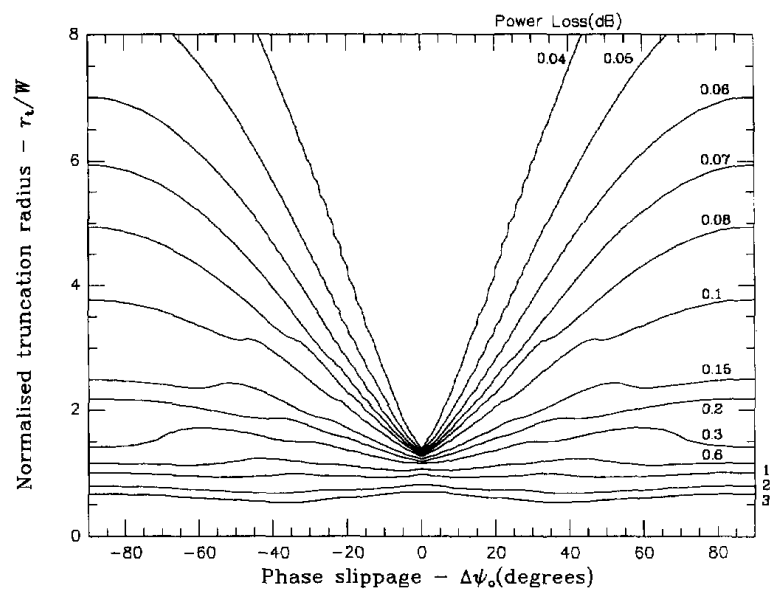

Fig. 2. Contours of constant power loss (dB) as a function of phase slippage and normalized truncation for the co-polar beam of a diagonal horn.

of the plot is required because the truncation loss is symmetric; conceptually, however, it is easier, when doing calculations, if both halves are visible.

In the case of the uniformly-illuminated aperture, it can be seen that there are poorly-defined sidelobes that propagate to high values of $r / W$ as $\Delta \psi_{0}$ increases. This structure is real and can be related to the number of Fresnel zones that fill the aperture. A similar structure appears in some of the other plots. It is clear from Fig. 4, that it is almost impossible to transmit the beam from a uniformly-illuminated aperture through an optical system: Beam spreading occurs even for small values of phase slippage, and away from the near-field region, a significant fraction of the power is diffracted to large values of $(r / W)$. At the other extreme, a corrugated horn only requires $r_{t} \geq 2.0 \mathrm{~W}$ for the losses to be less than $0.035 \mathrm{~dB}$, or equivalently $0.8 \%$. Fig. 1 confirms the rule of thumb

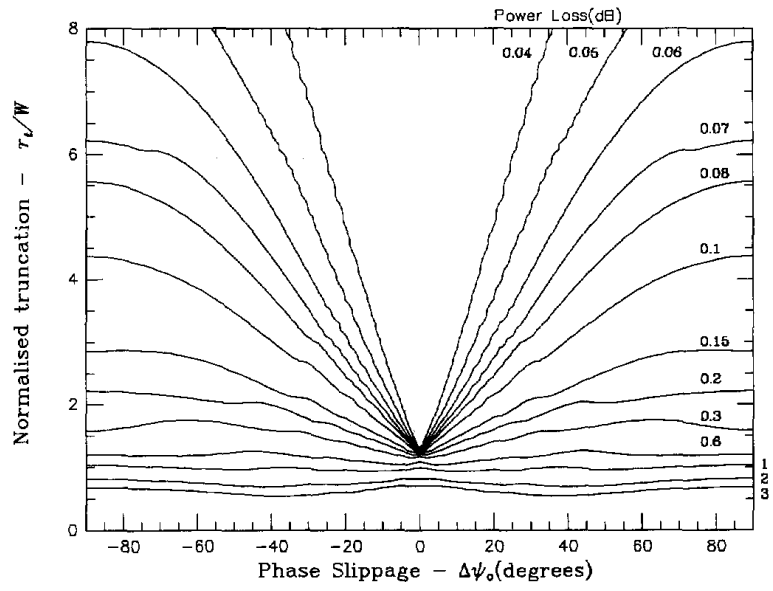

Fig. 3. Contours of constant power loss (dB) as a function of phase slippage and normalized truncation for the co-polar beam of a smoothwalled conical horn.

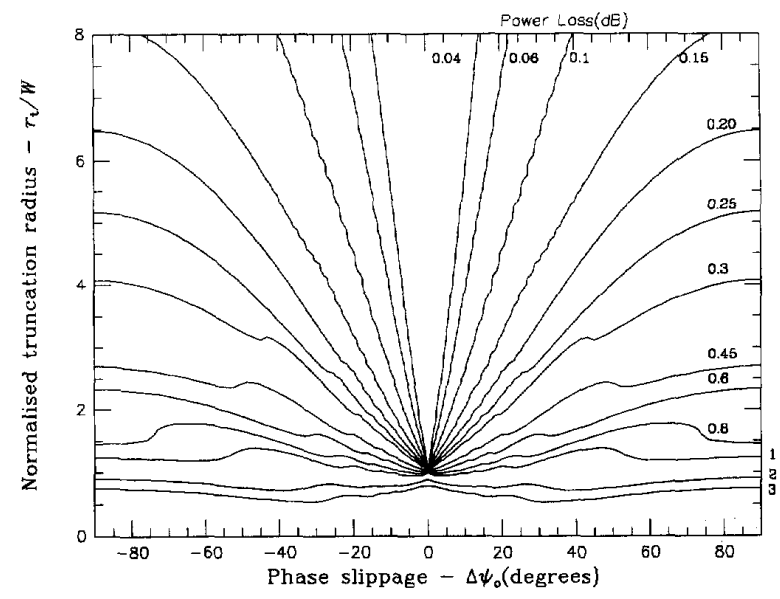

Fig. 4. Contours of constant power loss (dB) as a function of phase slippage and normalized truncation for the beam of a uniformly illuminated aperture.

that, for a corrugated horn, one has to use optical components that are larger than 3 beam radii. In certain regions of the system, however, the components can be very much smaller, and our graph quantifies this statement.

\section{Use of Contour Plots to Determine Power Loss}

In this section, we explain how to use the above plots to calculate the loss at any plane in a complicated optical system. We suggest the following procedure:

1. Determine the size of the beam at the aperture of the horn that maximises the power in the fundamental mode, so that $W_{h}=W_{h \text {, opt }}$. For the horns considered in this paper, the options are summarised in Table $I$.

2. Once $W_{h}$ is known, the position and size of the waist for the mode set chosen can be calculated. The distance 
TABLE II

Beam Parameters of an Optical System Comprising a Diagonal Horn, a LeNS aNd Two OfF-Axis MirRors. The Beam Parameters are Calculated at $400 \mathrm{GHz}$. The Horn is 19.0 MM LONG ANd has an APERTURE WHICH IS 3.5 MM SQUARE.

\begin{tabular}{llccccc}
\hline Component & Separation $(\mathrm{mm})$ & $W(\mathrm{~mm})$ & $e_{t} / W$ & $\Delta \psi(\mathrm{deg})$ & $P_{\mathrm{tr}}(\mathrm{dB})$ & Loss $(\%)$ \\
\hline Virtual waist & 3.8 & 1.35 & & -26 & & \\
Horn aperture & 32 & 1.5 & & 0 & & \\
Lens $(f=32)$ & 86 & 6.5 & 3.8 & 52 & 0.085 & 1.9 \\
Window $(50 \mathrm{~mm})$ & 280 & 5.1 & 4.9 & 90 & 0.075 & 1.8 \\
Mirror $(f=280)$ & 280 & 14.1 & 2.5 & -21 & 0.070 & 1.6 \\
Image & 350 & 13.2 & & 0 & & \\
Mirror $(f=350)$ & 350 & 14.7 & 2.4 & 25 & 0.085 & 1.9 \\
Cass focus & & 6.3 & & 90 & & \\
\hline
\end{tabular}

between the waist and the aperture is given by

$$
z_{h}=\frac{L}{1+\left(\lambda L / \pi W_{h}^{2}\right)^{2}}
$$

and the size of the waist is given by

$$
W_{0}=\frac{W_{h}}{\sqrt{1+\left(\pi W_{h}^{2} / \lambda L\right)^{2}}} .
$$

We also need the phase slippage between the waist and the aperture:

$$
\psi_{0}\left(z_{h}\right)=\arctan \left(\frac{z_{h} \lambda}{\pi W_{0}^{2}}\right)=\arctan \left(\frac{\pi W_{h}^{2}}{\lambda L}\right),
$$

where $L$ is the length of the horn.

3. Having calculated the position and size of the waist, propagate the beam through the optical system and calculate the size of the beam at the plane of interest, $W$ [1]. To complete the analysis one also needs the phase slippage between the aperture of the horn and the stop: $\Delta \psi_{0}(z)=\psi_{0}(z)-\psi_{0}\left(z_{h}\right)$, where $\psi_{0}(z)$ is the phase slippage between the waist and the stop. For example, if the truncating stop lies a distance $d$ in front of the aperture, then

$$
\begin{aligned}
\Delta \psi_{0}(z) & =\arctan \left(\frac{\lambda\left(d+z_{h}\right)}{\pi W_{0}^{2}}\right)-\arctan \left(\frac{\lambda z_{h}}{\pi W_{0}^{2}}\right) \\
& =\arctan \left(\frac{\lambda d L}{\pi W_{h}^{2}(d+L)}\right) .
\end{aligned}
$$

If there are intervening optical components, one can calculate the total phase slippage, by summing the phase slippages accumulated between each of the focusing elements. Obviously, when calculating an individual contribution, one must use the waist appropriate to that region.

4. Once the normalized radius $r_{t} / W$ and the phase slippage $\Delta \psi_{0}$ have been calculated, the appropriate contour plot can be used to determine the truncation loss. Note that if $\Delta\left(\psi_{0}\right)>\pi / 2$, then it should be rescaled by subtracting off an integer times $\pi$ so that $\Delta\left(\psi_{0}\right)-n \pi$ lies between $-\pi / 2$ and $\pi / 2$.

For example, consider the $200 \mathrm{GHz}$ to $900 \mathrm{GHz}$ optical system listed in Table II [12], where a diagonal horn is coupled to a reflecting antenna through a lens and two off-axis mirrors. The horn is part of a superconducting mixer which is located in a cryostat, and therefore the beam has to pass through a window which must be made as small as possible. In Table II, we list the phase slippage, normalized truncation radius, and truncation loss, for each of the main optical components.

It is clear from Fig. 2 that, for diagonal horns, it is difficult to clear the beam out to a loss better than $1.5 \%$. It is a mistake, however, to think that if one has a cascade of truncating components, the total loss is simply given by the sum of losses associated with the individual components. Obviously, if the beam does not diffract at any of the apertures, the total loss is determined by the aperture with the greatest loss, since it is not possible to lose power twice by truncating a beam at the same normalized radius twice. If the beam does diffract, then power can be lost twice. The above procedure should therefore be used as a way of establishing how big an aperture should be in order to avoid truncation. If one wants to calculate the total loss incurred in a system for which the diffraction at each component is significant, one must calculate how the beam spreads at each of the apertures. This calculation can be done by using a scattering matrix to characterize the way in which power is redistributed between modes as the beam passes through each stop [13], [14]. We have used a scattering-parameter technique to analyze the above optical system, and it turns out that, for this case, the total loss is, as expected, much less than the sum of the individual losses [15].

\section{CONCLUSIONS}

We have presented a technique for determining the power that is lost when the beam in a beam waveguide is truncated by an axially-symmetric stop. The technique is based on the notion that for a multimode Gaussian beam the scale size of the beam at a plane is characterized by 
the Gaussian radius, and the form of the beam is characterised by the phase slippage. Hence, the loss at a truncating aperture is completely determined by these two quantities also.

The most difficult part of an analysis lies in calculating contours of constant loss as a function of normalised truncation and phase slippage. If, however, the truncating aperture is circular, and the beam is expanded in terms of associated Laguerre modes, the necessary integration can be reduced to evaluating a recursion relationship.

Although, the underlying scheme is applicable to any long-focal system, we have concentrated on waveguides that are fed by diagonal horns, smooth-wall conical horns, corrugated horns, and uniformly-illuminated apertures; these antennas cover the whole range of behavior likely to be encountered in practice. We have calculated the appropriate contour plots for these horns, and we have shown how these plots can be used together with singlemode design techniques to minimize the size of complicated millimeter and submillimeter-wave optical systems.

\section{ACKNOWLEDGMENTS}

The authors would like to thank EOLAS and the British Council for financing travel.

\section{REFERENCES}

[1] P. F. Goldsmith, "Quasi-optical techniques at millimeter and submillimeter wavelengths," in Infrared and Millimeter Waves, vol. 8, pp. $277-343,1982$.

[2] R. J. Wylde, "Millimeter-wave Gaussian beam-mode optics and corrugated feed horns," Proc. Inst. Elec. Engrg., vol. 131, pt. H, pp. 258-262, 1984

[3] R. Padman, J. A. Murphy, and R. E. Hills, "Gaussian mode analysis of cassegrain antenna efficiency," IEEE Trans. Antennas Propagation, vol. AP-35, pp. 1093-1103, 1987.

[4] J. A. Murphy, "Aperture efficiencies of large axisymmetric reflector antennas fed by conical horns," IEEE Trans. Antennas Propagation, vol. AP-36, pp. 570-575, 1988.

[5] S. Withington and J. A. Murphy, "Analysis of diagonal horns through Gaussian-Hermite modes," IEEE Trans. Antennas Propagation, vol. AP-40, pp. 198-206, 1992.

[6] D. H. Martin and J. Lesurf. "Submillimeter Wave Optics," in Infrared Physics, vol. 10, pp. 105-109, 1978.

[7] J. W. Lamb, "Quasi-optical coupling of Gaussian beam systems to large Cassegrain antennas," Internat. J. Infrared Millimeter Waves, vol. 7, pp. 1511-1536, 1986

[8] H. Kogelnik and T. Li, "Laser beams and resonators," Proc. IEEE vol. 54, pp. 1312-1329, 1966.

[9] J. A. Murphy and S. Withington, "Truncation in beam waveguides," Proc. Fourth International Symposium on Space Terahertz Technology, University of California, pp. 184-198, April 1993.

[10] C. Aubry and D. Bittern, "Radiation patterns of a corrugated conical horn in terms of Laguerre-Gaussian Functions," Electron. Lett., vol. 11, pp. 154-156, 1975 .

[11] J. A. Murphy and R. Padman, "Phase centers of horn antennas using Gaussian beam mode analysis," IEEE Transactions Antennas Propagation, vol. AP-38, pp. 1306-1310, 1990.
[12] S. Withington, J. A. Murphy, A. Egan, and R. E. Hills. "On the design of broadband quasioptical systems for submillitre-wave radio-astronomy receivers," Internat. J. Infrared Millimeter Waves, vol. 13, pp. 1515-1537, 1992.

[13] P. Belland and J. P. Crenn. "Changes in the characteristics of a Gaussian beam weakly diffracted by a circular aperture," Appl. Optics, vol. 21, pp. 522-527, 1982.

[14] R. Padman and J. A. Murphy, "A scattering matrix formulation for Gaussian beam mode analysis," Proceedings of the IEE /URSI 7th Int. Conference on Antennas and Propagation, ICAP, pp. 201-204, York, April, 1991

[15] J. A. Murphy, S. Withington, and A. Egan, "Mode-conversion at diffracting apertures in millimetre and submillitre-wave optical systems." Accepted for publication in the IEEE Trans. Mircowave Theory Technol.

J. Anthony Murphy was born in Cork, Ireland, on October 30, 1954. $\mathrm{He}$ received the B.Sc. and M.Sc. degrees in Experimental Physics from University College Cork, Ireland, in 1977 and 1979, respectively, the M.S. degree in Physics from the California Institute of Technology in 1981 and the Ph.D. in Physics from the University of Cambridge, England, in 1986.

From 1985 to 1987 he was a Research Associate at the Cavendish Laboratory, Cambridge, where he was engaged in receiver development for the James Clerk Maxwell Telescope on Mauna Kea, Hawaii. In 1988 he was appointed as lecturer in the Experimental Physics Department, St. Patrick's College, Maynooth, Ireland. His main research interest is in millimeter and submillimeter-wave optics. For a photo please see page 206 of the February 1992 issue of this journal.

Stafford Withington was born in Chesterfield, England, on May 27, 1957. He received the B.Eng.Hons degree, first class, from the University of Bradford, England, in 1979, and the Ph.D. degree in Radio Astronomy from the University of Manchester, England, in 1983.

$\mathrm{He}$ has held a number of positions, including a lectureship in the Department of Electrical Engineering at Sheffield University and a Royal Society Overseas Fellowship in the Department of Radio and Space Science at Chalmers University of Technology, Sweden. He is currently a Senior Research Associate in the Department of Physics at Cambridge University and a Fellow of Downing College, Cambridge. In recent years he has concentrated on the development of low-noise submillimeter-wave receivers for use in radio astronomy, and he is particularly interested in superconducting detector technology and submillimeter-wave optics. For a photo please see page $\mathbf{2 0 6}$ of the February 1992 issue of this journal.

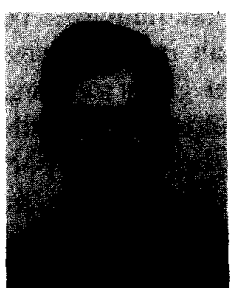

Aidan Egan was born in Offaly, Ireland, on April 30, 1969. He received the B.Sc. and M.Sc degrees in Physics from St. Patrick's College, Maynooth, Ireland, in 1990 and 1992, respectively. Currently he is pursuing the Ph.D. degree in Physics at Trinity College, Dublin, Ireland, where his research interests are in modelling of multielectrode semiconductor lasers, self-pulsation and optical synchronisation. 